\title{
Consumo de bebidas alcoólicas em estudantes de enfermagem de um centro universitário
}

\author{
Márcia Astrês Fernandes ${ }^{1}$ \\ Frankiana Medeiros Moita ${ }^{2,3}$ \\ Marcelo Pereira Vieira Nascimento ${ }^{2}$ \\ José Diego Marques Santos ${ }^{1}$ \\ Miguel Henrique Pereira de Paiva ${ }^{4}$
}

${ }^{1}$ Universidade Federal do Piauí, Teresina, PI, Brasil.

${ }^{2}$ Centro Universitário Uninovafapi, Teresina, PI, Brasil.

${ }^{3}$ Maternidade Dona Evangelina Rosa, Teresina, PI, Brasil.

${ }^{4}$ Faculdade Integral Diferencial, Teresina, PI, Brasil.
Objetivo: investigar o padrão de consumo de álcool em estudantes de enfermagem de um Centro Universitário. Métodos: trata-se de um estudo exploratório, descritivo, com abordagem quantitativa, desenvolvido com 372 estudantes de enfermagem de um Centro Universitário. Os dados foram coletados no mês de maio de 2014, mediante aplicação de um questionário sociodemográfico e da escala de AUDIT. Resultados: evidenciou-se que $90,6 \%$ dos acadêmicos usam ou já fizeram uso de bebida alcoólica. Quando avaliado o resultado por zona do AUDIT, evidenciou-se que a maioria dos entrevistados 162 (44\%) foi classificada na Zona II. Conclusão: torna-se relevante a condução de futuras pesquisas para desvendar fatores de proteção e de risco para o uso de álcool.

Descritores: Alcoolismo; Consumo de Bebidas Alcoólicas; Estudantes de Enfermagem; Saúde Mental.

\section{Como citar este artigo}

Fernandes MA, Moita FK, Nascimento MPV, Santos JDM, Paiva MHP. Alcohol consumption among nursing students of a university center. SMAD, Rev Eletrônica Saúde Mental Álcool Drog. 2019;15(2):38-44. doi: https://dx.doi.org/10.11606/issn.1806-6976.smad.2019.000401 


\title{
Alcohol consumption among nursing students of a university center
}

\begin{abstract}
Objective: to investigate the pattern of alcohol consumption in nursing students at a University Center. Methods: this is an exploratory, descriptive study with a quantitative approach, developed with 372 nursing students from a University Center. Data were collected in May 2014, using a socio-demographic questionnaire and the AUDIT scale. Results: it was evidenced that $90.6 \%$ of the students use or have already used alcohol. When evaluating the result by area of the AUDIT, it was evidenced that the majority of respondents 162 (44\%) was classified in Zone II. Conclusion: it is relevant to conduct future research to uncover protection and risk factors for
\end{abstract} alcohol use.

Descriptors: Alcoholism; Alcohol Drinking; Nursing Students; Mental Health.

\section{Consumo de bebidas alcohólicas en estudiantes de enfermería de un centro universitario}

Objetivo: investigar el patrón de consumo de alcohol en estudiantes de enfermería de un Centro Universitario. Métodos: se trata de un estudio exploratorio, descriptivo, con abordaje cuantitativo, desarrollado con 372 estudiantes de enfermería de un Centro Universitario. Los datos fueron recolectados en el mes de mayo de 2014, mediante la aplicación de un cuestionario socio demográfico y de la escala de AUDIT. Resultados: se evidenció que el 90,6\% de los académicos usan o ya han hecho uso de bebidas alcohólicas. Cuando se evaluó el resultado por zona del AUDIT, se evidenció que la mayoría de los entrevistados 162 (44\%) fue clasificada en la Zona II. Conclusión: se hace relevante la conducción de futuras investigaciones para desvelar factores de protección y de riesgo para el uso de alcohol.

Descriptores: Alcoholismo; Consumo de Bebidas Alcohólicas; Estudiantes de Enfermería; Salud Mental. 


\section{Introdução}

O álcool é consumido por grande parte das pessoas na maioria dos países do mundo, possuindo grande aceitação social, e seu consumo, estimulado pela sociedade. O uso é cada vez mais frequente apesar dos riscos à saúde(1).

Mundialmente, cerca de $38,3 \%$ da população maior de 15 anos consome bebidas alcoólicas. Nesse mesmo grupo, o consumo de álcool per capita chega a 17,2 litros (WHO, 2014). Além disso, entre as pessoas com idades de 15 a 49 anos, o abuso de álcool é o principal fator de risco para morte prematura e incapacidade. $\mathrm{Na}$ faixa etária 20-39 anos, aproximadamente $25 \%$ do total de mortes são atribuídas ao mesmo fator ${ }^{(2-3)}$.

Dados sobre o consumo de álcool em estudantes de enfermagem ainda são pouco conhecidos; no entanto, fatores relacionados à predisposição para o consumo de bebidas alcoólicas nessa população têm sido discutidos, sendo os principais: falta de orientação, expectativas sobre o curso com aumento do estresse, influências ambientais e individuais e disponibilidade de álcool relacionada às políticas ineficazes ${ }^{(4-5)}$.

As consequências negativas do consumo de álcool em acadêmicos são incontáveis e estão relacionadas à problemas biopsicossociais em curto e longo prazo e acidentes por causas externas intencionais ou não intencionais (acidentes automobilísticos e violência, principalmente), além de déficits no desempenho acadêmico(6-8). Nesse contexto, dada a relevância do assunto, o objetivo deste estudo foi investigar o padrão do consumo de álcool em estudantes de enfermagem de um Centro Universitário.

\section{Métodos}

Tratou-se de um estudo quantitativo, descritivo e exploratório. O local da realização deste estudo foi um centro universitário do nordeste do Brasil, instituição de ensino superior privada e credenciada junto ao Ministério da Educação.

Em relação à população do estudo, 570 acadêmicos encontravam-se devidamente matriculados no curso de enfermagem da referida instituição. Como critérios de inclusão, adotaram-se: ser aluno do curso de Enfermagem do centro universitário e estar ativo no curso independentemente do período. Como critério de exclusão, elegeu-se: não estar presente no momento da coleta de dados.

Para calcular o tamanho da amostra, optou-se pelo cálculo das populações finitas, considerando um intervalo de confiança de $95 \%$ e erro amostral tolerável de $3 \%$, chegou-se a um quantitativo de 372 participantes.

Os dois instrumentos foram aplicados no próprio local da pesquisa em horário pré-estabelecido de modo que não viesse a interferir nas atividades acadêmicas. A fim de preservar o anonimato dos participantes, uma caixa foi colocada em locais pré-estabelecidos para que os questionários pudessem ser depositados após o preenchimento.

A coleta de dados foi realizada por meio do preenchimento de dois questionários: um sociodemográfico, levando em consideração as variáveis do estudo e o outro instrumento foi o Teste para Identificação de Problemas relacionados ao uso do Álcool-AUDIT, que avalia o padrão de uso e o comportamento nos últimos 12 meses.

A partir dos dados obtidos, foi criado um banco de dados no Programa Microsoft Excel 2010, sendo posteriormente exportado para o Statistical Package for Social Sciences(SPSS) 20.0 para Windows, com isso processado e analisado estatisticamente. A variável dependente do estudo foi o uso de álcool, classificada como dicotômica (sim/não).

As variáveis quantitativas mensuradas por meio de média e desvio-padrão foram: idade de início do uso, tempo de uso e frequência utilizada. As variáveis qualitativas foram: escolaridade, situação conjugal, renda familiar, ocupação, religião, procedência, local do primeiro consumo, oferta do consumo, local de maior frequência, tipo de bebida mais utilizada e interferência na vida cotidiana. Os resultados foram apresentados em tabelas e gráficos.

A participação no estudo foi voluntária, mediante leitura e assinatura de Termo de Consentimento Livre e Esclarecido, fundamentado no capítulo IV, da Resolução 466/2012 do Conselho Nacional de Saúde, podendo estes participantes desvincularem-se da pesquisa a qualquer momento, sem prejuízos ou qualquer ônus. Assim, foi garantido a cada participante o sigilo das informações obtidas individualmente, enfatizando quanto ao anonimato do questionário aplicado em sala de aula.

O Projeto foi aprovado pelo Comitê de Ética em Pesquisa do Centro Universitário UNINOVAFAPI, sob Certificado de Apresentação para Apreciação Ética (CAAE) -30353214.6.0000.5210. Todos os procedimentos éticos e legais foram respeitados, em conformidade com a Resolução 466/2012 do Conselho Nacional de Saúde (CNS).

\section{Resultados}

A análise dos resultados possibilitou categorizar as informações de acordo com as características sociais e demográficas, perfil do consumo, frequência e a distribuição do consumo de bebidas alcoólicas entre acadêmicos de enfermagem.

Identificou-se que a maioria dos indivíduos participantes deste estudo cursava o $6^{\circ}$ período do curso 
$(20,7 \%)$, eram solteiros $(73,1 \%)$, com renda familiar de 1-3 SM (54,8\%), sem ocupação $(91,7 \%)$, católico $(62,6 \%)$, e residente em Teresina $(53,8 \%)$ (Tabela 1$)$.

Tabela 1 - Caracterização sociodemográfica dos estudantes de enfermagem de um Centro Universitário. Teresina, Piauí, Brasil, $2014(n=372)$

\begin{tabular}{|c|c|c|}
\hline & N & $\%$ \\
\hline \multicolumn{3}{|l|}{ Período do curso } \\
\hline $1^{\circ}$ período & 45 & 12,1 \\
\hline $2^{\circ}$ período & 48 & 12,9 \\
\hline $3^{\circ}$ período & 37 & 9,9 \\
\hline $4^{\circ}$ período & 40 & 10,8 \\
\hline $5^{\circ}$ período & 33 & 8,9 \\
\hline $6^{\circ}$ período & 77 & 20,7 \\
\hline $7^{\circ}$ período & 40 & 10,8 \\
\hline $8^{\mathrm{a}}$ período & 52 & 14,0 \\
\hline \multicolumn{3}{|l|}{ Estado civil } \\
\hline Solteiro (a) & 272 & 73,1 \\
\hline Casado (a) & 35 & 9,4 \\
\hline Viúvo (a) & 03 & 0,8 \\
\hline Outros & 62 & 16,7 \\
\hline \multicolumn{3}{|l|}{ Renda familiar } \\
\hline Até $1 \mathrm{SM}$ & 62 & 16,7 \\
\hline $1-3 \mathrm{SM}$ & 204 & 54,8 \\
\hline 4-6 SM & 58 & 15,6 \\
\hline$>6 \mathrm{SM}$ & 48 & 12,9 \\
\hline \multicolumn{3}{|l|}{ Ocupação } \\
\hline Sim & 31 & 8,3 \\
\hline Não & 341 & 91,7 \\
\hline \multicolumn{3}{|l|}{ Religião } \\
\hline Católica & 233 & 62,6 \\
\hline Espirita & 46 & 12,4 \\
\hline Evangélico & 76 & 20,4 \\
\hline Nenhuma & 15 & 4,0 \\
\hline Outro & 02 & 0,5 \\
\hline \multicolumn{3}{|l|}{ Procedência } \\
\hline Teresina & 200 & 53,8 \\
\hline Interior do estado & 125 & 33,6 \\
\hline Outros & 47 & 12,6 \\
\hline
\end{tabular}

Considerando-se a variável dependente "uso de álcool", classificada como ( $\operatorname{sim} /$ não), verificou-se que $90.6 \%$ ( $n=337$ ) dos acadêmicos usam ou já fizeram uso de bebida alcoólica, enquanto que apenas 9,4\% $(n=35)$ nunca consumiram bebida alcoólica.

A faixa etária média dos participantes foi de 17,5 anos de idade $(D P=2,0)$. Já em relação ao tempo de uso e frequência no consumo de álcool, obtiveram-se resultados variados, com a maioria dos estudantes $(58,9 \%)$ fazendo uso esporádico de bebidas alcoólicas (Figura 1).
Início de uso e frequência de uso por semana

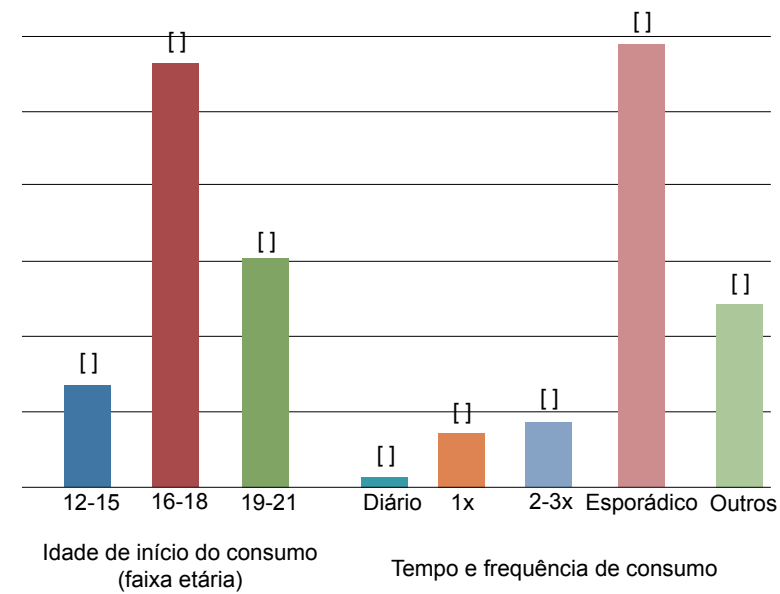

Figura 1 - Padrão de consumo de álcool (quantidade e frequência) realizado por estudantes de enfermagem de um Centro Universitário quanto à frequência, idade de início de uso. Teresina, Piauí, Brasil, 2014. $(n=372)$

Detectou-se que a maioria dos indivíduos pesquisados consumiu álcool pela primeira vez na casa de amigos $(49,7 \%)$, com oferta de amigos $(53,8 \%)$, em bares ou boates $(49,7 \%)$, prevalecendo o uso da cerveja $(54,8 \%)$, e sem interferência na vida cotidiana $(61,8 \%)$ (Tabela 2).

Tabela 2 - Características relacionadas ao consumo e tipo de bebida alcoólica consumida por estudantes de enfermagem de um Centro Universitário. Teresina, Piauí, Brasil, 2014

\begin{tabular}{lcc}
\hline & N & $\%$ \\
\hline Local do primeiro consumo & & \\
Em casa & 138 & 37,1 \\
Bares/Boates & 33 & 8,9 \\
Casa de amigos & 185 & 49,7 \\
Outros & 15 & 4,0 \\
Não recordo & 01 & 0,3 \\
Oferta de consumo & & \\
Amigos & 200 & 53,8 \\
Familiares & 35 & 9,4 \\
Sozinho & 20 & 5,4 \\
Parceiro/companheiro & 16 & 4,3 \\
Local de maior frequência & & \\
Em casa & 55 & 14,8 \\
Bares/Boates & 185 & 49,7 \\
Casa de amigos & 99 & 26,6 \\
Outros & 36 & 9,7 \\
Tipo de bebida & & \\
Cerveja & 204 & 54,8 \\
Cachaça & 06 & 1,6 \\
Uísque & 46 & 12,4 \\
Rum & 17 & 4,6 \\
Vodca & 29 & 7,8 \\
Tequila & 21 & 5,6 \\
Vinho & 30 & 8,1 \\
Outros & 07 & 1,9 \\
Mais de um tipo & 12 & 3,2 \\
\hline & & $($ continua $\ldots)$ \\
& &
\end{tabular}


Tabela 2 - continuação

\begin{tabular}{lcc}
\hline & N & $\%$ \\
\hline Interferência na vida cotidiana & & \\
$\quad$ Nunca & 230 & 61,8 \\
Menos de 1 vez por mês & 95 & 25,5 \\
Pelo menos 1 vez por mês & 04 & 1,1 \\
Pelo menos 1 vez por & 04 & 1,1 \\
semana & 03 & 0,8 \\
$\quad$ Diariamente ou quase & 03 \\
\hline
\end{tabular}

Quando avaliado o resultado por zona do AUDIT, evidenciou-se que a maioria dos entrevistados 162 (44\%) foi classificada na Zona II, ou seja, tem um consumo de bebidas alcoólicas indicado como uso de risco (Figura 2).

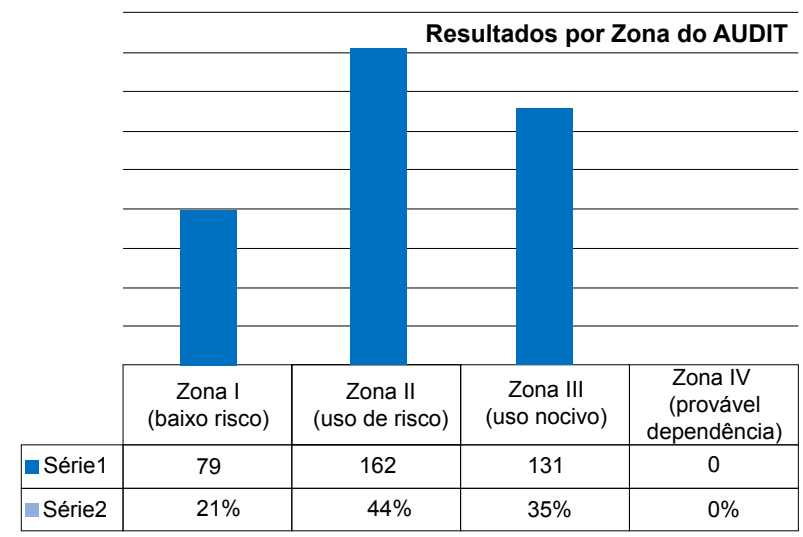

Figura 2 - Classificação dos participantes por zona AUDIT. Teresina, Piauí, Brasil, 2014

\section{Discussão}

Os resultados deste estudo apontaram que mais de $90 \%$ dos acadêmicos de enfermagem de um centro universitário já consumiram algum tipo de bebida alcoólica. Tal porcentagem supera de sobremaneira aquela apresentada por estudos realizados em outras regiões do país(9-11), um achado que merece atenção.

Sabe-se, ainda, que há diversos fatores relacionados ao consumo de álcool na população. Dentre eles estão a idade, gênero, fatores familiares, status socioeconômico, contexto cultural e regulações sobre o uso(12-13). Esse fato explica a variação nas taxas de prevalência no uso de álcool entre acadêmicos de enfermagem em diferentes estudos.

Pesquisa conduzida em município de Rondônia-RO identificou a prevalência de consumo de álcool em 58\% dos 137 estudantes de enfermagem investigados ${ }^{(11)}$. Em Minas Gerais, estudos similares observaram prevalência de $65,5 \%$ em uma amostra de 168 estudantes de enfermagem(10), e de $81,7 \%$ em uma amostra de $191^{(9)}$.

A iniciação do uso de álcool pela maioria $(56,18 \%)$ dos participantes da pesquisa deu-se antes ou imediatamente depois de atingida a maioridade, na faixa etária entre 16 e 18 anos de idade. Estudo epidemiológico descritivo do tipo transversal desenvolvido em uma universidade privada da cidade do Rio de Janeiro, Brasil, com 161 discentes de enfermagem, revelou um dado ainda mais preocupante, apontando que, em $85 \%$ dos 109 indivíduos entrevistados, o início deu-se antes dos 18 anos $^{(14)}$.

Em relação à frequência do consumo de álcool, a maioria dos indivíduos pesquisados $(58,87 \%)$ referiu uso esporádico, não bebendo semanalmente. Mesmo consumido de forma moderada e esporadicamente, o álcool não deixa de trazer malefícios, dentre os quais se destacam acidentes (intencionais ou não intencionais) e comportamento de risco, principalmente entre os jovens ${ }^{(15)}$. Além disso, evidências apontam que o consumo de álcool venha a ser um agente motivador à dependência ou mesmo ao consumo de outras drogas ${ }^{(11)}$.

Sobre o perfil sociodemográfico da amostra estudada, os dados deste estudo convergem com o de uma pesquisa realizada com 404 estudantes do curso de licenciatura de enfermagem em Portugal, no qual se observou uma média de idade de aproximadamente $20 \operatorname{anos}^{(16)}$. Sabe-se que o álcool está entre as drogas mais usadas para a população jovem e seu uso pode ser desencadeado por rebeldia, curiosidade e grande influência de amizades ${ }^{(17)}$.

Pesquisa realizada em Teresina-PI, com acadêmicos do curso de graduação em Enfermagem de uma instituição de ensino superior identificou resultados semelhantes para o estado civil, a procedência e a ocupação(18). Desse modo, percebe-se que o estudante de enfermagem depende de suporte familiar, sobretudo quando se trata de aspectos financeiros, para que possa arcar com suas despesas em geral.

Evidenciou-se que a maioria dos participantes era católica. Um dado similar foi encontrado em uma pesquisa realizada com 167 discentes de Enfermagem da Universidade Estadual de Montes Claros, MG, no qual $61,7 \%$ referiram ser católicos(19). A dimensão religiosa, quando presente no cotidiano dos indivíduos, sobretudo entre os jovens, pode representar um fator influenciador para as relações sociais e familiares, apoiados por pressupostos éticos, políticos e culturais. Algumas práticas religiosas são capazes de proporcionar aspectos salutares que implicam positivamente na saúde física e mental à medida que aconselham a adoção de hábitos e condutas saudáveis. Pesquisa realizada com 6.264 estudantes brasileiros do ensino médio revelou que possuir uma religião é um fator de proteção para o uso de álcool e outras drogas(20).

A religião oferece uma fonte de força pessoal para o indivíduo, uma orientação de vida mais otimista e maior resiliência ao estresse e menos ansiedade, 
favorecendo uma ótica positiva frente ao enfrentamento do tratamento de usuários, bem como na proteção ao uso abusivo de álcool(21).

O local de consumo de álcool diverge em parte de um estudo entre universitários da área da saúde da Universidade Federal do Rio Grande/RS, que apontou que $70,2 \%$ consumiam em festas e churrascos, $24,5 \%$ na própria moradia e $4,8 \%$ em bares e restaurantes próximos à universidade(22).

Chama atenção nesta pesquisa o consumo de álcool no domicílio (14,8\%). O consumo de álcool pode ser iniciado no ambiente familiar, na idade entre 12 e 13 anos. Considera-se a adolescência como uma fase em que se dá bastante importância aos grupos de pertencimento, tornando o indivíduo mais vulnerável à influência dos outros na aquisição de comportamentos de risco, porém, são os valores e as atitudes adotadas pelos pais os norteadores da conduta dos filhos, oferecendo proteção ou risco para os jovens, inclusive para o consumo de álcool(23).

Na presente pesquisa, merece destaque a oferta de consumo pelos amigos (53,8\%). Relacionado a isso, há quem afirme que não ter amigos é um fator de proteção para uso de álcool, especificamente ${ }^{(24)}$. Uma pesquisa com 5521 jovens suíços, com cerca de 20 anos de idade, investigou a pressão de amigos para o uso de álcool e identificou esta variável como um fator de risco(25).

A prevalência do consumo de cerveja (54,8\%) entre os participantes pode ser explicada pelo fato de a mesma ser considerada uma "bebida leve", cuja iniciação já se apresenta no seio familiar(22). No Brasil, a propaganda das cervejas é livre, pois a mesma não é incluída como bebida alcoólica pela legislação vigente, sob o argumento do "baixo teor alcoólico"(8).

Ao se tratar do padrão do uso de álcool pelos estudantes desta pesquisa, evidencia-se que os achados vão ao encontro de um estudo que aplicou o AUDIT com 404 universitários dos cursos de Bioquímica, Enfermagem, Farmácia e Medicina. Esse estudo identificou que $46,0 \%$ dos participantes estavam com uso de risco e $24,3 \%$ com uso nocivo(26).

Esse tipo de consumo pode não ser presente em outras realidades. Em uma pesquisa realizada com estudantes de enfermagem da Universidade Castilla-La Mancha, Espanha, a prevalência de uso nocivo foi de $43,4 \%{ }^{(27)}$. O uso nocivo de álcool pode ser o pontapé inicial para a dependência; nesse trâmite, o usuário pode apresentar transtornos mentais ou hiperatividade psicomotora. A dependência também acarreta em alteração ou déficit no funcionamento social e familiar. Efeitos deletérios na saúde envolvendo padrão de sono, sexualidade e nutrição também podem ser encontrados(28).

O uso de álcool costuma chamar atenção nas populações jovens, sobretudo quando se trata de estudantes universitários. O estresse acadêmico, comumente imbricado à competitividade, esgotamento físico e mental decorrente de atividades universitárias, pode desempenhar o papel de conduzir os alunos a encontrarem no uso do álcool uma forma de

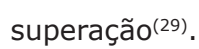

Embora relevante, o estudo apresenta limitação por se reportar a um cenário local, não permitindo, assim, generalizações. Desse modo, torna-se importante a realização de novas investigações com abrangência geográfica mais ampla.

\section{Conclusão}

Espera-se que o esclarecimento do contexto evidenciado por essa pesquisa sensibilize não somente os universitários quanto à importância da manutenção do seu equilíbrio físico e mental, mas também as coordenações pedagógicas, a fim de promover a amplificação da discussão sobre o tema e a elaboração de intervenções preventivas ao consumo de substâncias psicoativas, em especial o álcool, no meio acadêmico.

Chama-se também a atenção para a necessidade da condução de pesquisas que avaliem os fatores de risco e de proteção para o uso de álcool e outras drogas, considerando que são valiosos para o planejamento e implementação de medidas que corroborem para a redução das prevalências encontradas. Ademais, sugere-se um maior investimento em políticas públicas preventivas direcionadas para a população estudantil universitária.

\section{Referências}

1. Nimtz MA, Tavares AMF, Maftum MA, Ferreira ACZ, Capistrano FC. Legal and labor impacts on chemichal dependets' lives. Rev Eletrôn Saúde Mental Álcool Drog. 2016;12(2):68-74. doi: 10.11606/issn.1806-6976. v12i2p68-74

2. Lim SS, Vos T, Flaxman AD, Danaei G, Shibuya K, Adahir- $R$, et al. A comparative risk assessment of burden of disease and injury attributable to 67 risk factors and risk factor clusters in 21 regions, 1990-2010: a systematic analysis for the Global Burden of Disease Study 2010. Lancet. 2012;380(9859):2224-60.

3. World Health Organization (WHO). Alcohol. Geneva: WHO; 2015.

4. Nair JM, Nemeth LS, Sommers M, Newman S. Substance abuse policy among nursing students: a scoping review. J Addict Nurs. 2015;26(4)166-74.

5. Nair JM, Nemeth LS, Sommers M, Newman S, Amella E. Alcohol use, misuse, and abuse among nursing students: a photovoice study. J Addict Nurs. 2016;27(1):12-23. 
6. Centers for Disease Control and Prevention (CDCP). Fact Sheets - Alcohol Use and Your Health. Washington: CDCP; 2016.

7. Hakucho A, Kawamura H, Liu J, Liu X, Takase I, Fujimiya T. Relationship between alcohol consumption and external causes of death based on the forensic autopsy cases in Yamaguchi. Nihon Arukoru Yakubutsu Igakkai Zasshi. 2014;49(3):177-87.

8. Malta DC, Berna RTI, SIlva MMA, Claro RM, Silva Júnior JB, Reis AAC. Consumo de bebidas alcoólicas e direção de veículos, balanço da lei seca, Brasil 2007 a 2013. Rev Saúde Pública. 2014;48(4):692-6.

9. Pillon SC, Santos MA, Gonçalves AMS, Araújo KM. Alcohol use and spirituality amongnursing students. Rev Esc Enferm USP. 2011;45(1):98-105.

10. Marques NFB, Maciel EAF, Barbosa FI. Alcohol consumption by nursing students of a higher education institution. Rev Enferm Centro Oeste Paulista. 2012;2(2):159-65.

11. Vale JS, Uesugui HM, Pereira RA. Profile of consumption of alcohol, tobacco and marijuana among nursing students of Faculdade de Educação e Meio Ambiente (FAEMA). Rev Cient Fac Educ Meio Ambiente. 2014;5(2):156-72.

12. World Health Organization (WHO). Global status report on alcohol and health 2014. Geneva: WHO; 2014.

13. Morales GA, Galera SAF, Reyes AT, Aguila SRG, Arroyo MLA, Castillo FAM. Risk factors for alcohol consumption in adolescents students. SMAD, Rev. Eletrônica Saúde Mental Álcool Drog. 2017;13(1):22-9.doi: 10.11606/issn.18066976.v13i1p22-29

14. Tavares-Jomar R, Silva ES. Consumption of Alcoholic Beverages among Nursing Students. Aquichán. [Internet] 2013 [cited Dec 07 2018];13(2):226-33. Available from: <http://www.scielo.org.co/scielo.php?script=sci_arttext \&pid $=$ S1657-59972013000200009\&lng =en\&nrm =iso $>$. ISSN 1657-5997.

15. Cardoso LGV, Melo APS, Cesar CC. Prevalence of moderate and excessive alcohol consumption and associated factors among residents of Quilombo Communities (hinterland settlements founded by people of African origin) of Vitória da Conquista, Bahia, Brazil. Ciênc Saúde Coletiva. 2016;20(3):809-20.

16. Chaves C, Nelas P, Cruz C. Coutinho E, Amaral O. $\mathrm{O}$ perfil sociodemográfico e acadêmico dos estudantes de enfermagem e o bem estar. Psicol Ciênc Prof. 2016;1(2):123-32.

17. Lobo LA, Barbosa MCL. Álcool e drogas: um problema vivido por adolescentes usuários em um município do sudoeste da Bahia. Id on Line Rev Psic. 2017;10(33):32-42.
18. Oliveira LB, Nunes BMVT, Mourão LF. Perfil sociodemográfico do discente do curso de graduação em enfermagem. Rev Enferm UFPI. 2015;4(1):33-9.

19. Lima CA, Vieira MA, CostaFM. Caracterização dos estudantes do curso de graduação em Enfermagem de uma universidade pública. RENOME. 2014;3(2):33- 46.

20. Santos ARM, Oliveira LMFT, Farias JJC, Silva PPC, Silva EAPC, Freitas CMSM. Association between religious practice and health risk behaviors in adolescents of northeast, Brazil. Rev Bras Ativ Fis Saúde. 2015;20(3):284-96.

21. Oliveira ALCB, Feitosa CDA, Santos AG, Lima LAA, Fernandes MA, Monteiro CFS. Spirituality and religiosity in the context of drug abuse. Rev Rene. 2017;18(2):283-90. 22. Baumgarten LZ, Gomes VLO, Fonseca AD. Consumo alcoólico entre universitários(as) da área da saúde da Universidade Federal do Rio Grande/RS: subsídios para a enfermagem. Esc Anna Nery. 2012;16(3)530-5.

23. Elicker E, Palazzo LS, Aerts DRGC, Alves GG, Câmara S. Use of alcohol, tobacco and other drugs by adolescent students from Porto Velho-RO, Brazil. Epidemiol Serv Saúde. 2015;24(3):1-12.

24. Malta DC, Oliveira-Campos M, Prado RR, Andrade SSC, Mello FCM, Dias AJR, et al. Psychoactive substance use, family context and mental health among Brazilian adolescents, National Adolescent School-based Health Survey (PeNSE 2012). Rev Bras Epidemiol Suppl. 2014;1(1)46-61.

25. Studer J, Baggio S, Deline S, N'Goran AA, Henchoz Y, Moler-Kuo $M$, et al. Peer pressure and alcohol use in young men: a mediation analysis of drinking motives. Int J Drug Policy. 2014;24(4):700-8.

26. Carneiro ALM, Rodrigues SB, Gherardi-Donato ECS, Guimarães EAA, Oliveira VC. Padrão de uso de álcool entre estudantes universitários da área da saúde. Rev Enferm Centro Oeste Min. 2014;4(1)940-50.

27. Sotos JR, Gonzales AL, Martínez IP, Rosa MA, Herrarez MJS, Hidalgo JL. Prevalence of hazardous drinking among nursing students. J Adv Nurs. 2015;71(3):581-90.

28. Cruz ACM, Lisboa AR, Sousa JBG, Leite FC. O uso do álcool e suas consequências na saúde dos consumidores. Rev FAMA Cienc Saúde. 2015;1(2):11-8.

29. Hirsch CD, Barlem ELD, Almeida LK, TomaschewskiBarlem JG, Figueira AB, Lunardi VL. Coping strategies of nursing students for dealing with university stress. Rev Bras Enferm. 2015;68(5):501-8. 